Article

\title{
Multi-Objective Optimal Design of Permanent Magnet Synchronous Motor for Electric Vehicle Based on Deep Learning
}

\author{
Yong-min You 1 \\ Department of Automotive Engineering, Honam University, Gwangju 62399, Korea; ym.you@honam.ac.kr; \\ Tel.: +82-62-940-5499
}

Received: 5 December 2019; Accepted: 3 January 2020; Published: 9 January 2020

Featured Application: Optimal design of PMSM for electric vehicle.

\begin{abstract}
Recently, a large amount of research on deep learning has been conducted. Related studies have also begun to apply deep learning techniques to the field of electric machines, but such studies have been limited to the field of fault diagnosis. In this study, the shape optimization of a permanent magnet synchronous motor (PMSM) for electric vehicles (EVs) was conducted using a multi-layer perceptron (MLP), which is a type of deep learning model. The target specifications were determined by referring to Renault's Twizy, which is a small EV. The average torque and total harmonic distortion of the back electromotive force were used for the multi-objective functions, and the efficiency and torque ripple were chosen as constraints. To satisfy the multi-objective functions and constraints, the angle between the V-shaped permanent magnets and the rib thickness of the rotor were selected as design variables. To improve the accuracy of the design, the design of experiments was conducted using finite element analysis, and a parametric study was conducted through analysis of means. To verify the effectiveness of the MLP, metamodels was generated using both the MLP and a conventional Kriging model, and the optimal design was determined using the hybrid metaheuristic algorithm. To verify the structural stability of the optimized model, mechanical stress analysis was conducted. Moreover, because this is an optimal design problem with multi-objective functions, the changes in the optimal design results were examined as a function of the changes in the weighting. The optimal design results showed that the MLP technique achieved better predictive performance than the conventional Kriging model and is useful for the shape optimization of PMSMs.
\end{abstract}

Keywords: deep learning; finite element analysis; PMSM; optimization; metamodeling

\section{Introduction}

Optimal design is a method of finding the values of design variables to obtain an optimal solution within a range of constraints. Owing to the developments in design technology and improvements in computational ability, the optimal design method has been applied to solve complex design problems in various industrial fields. Many studies have been conducted on the optimal design of permanent magnet synchronous motors (PMSMs), the heart of electric vehicles (EVs), which have rapidly grown in popularity in recent years. This is because PMSMs for EVs must satisfy various goal specifications simultaneously, including torque and efficiency.

The optimal design for a PMSM can be created by combining design methods such as analytical models [1,2], magnetic equivalent circuit (MEC) models [3,4], and finite element analysis (FEA) [5-8] with an optimal design algorithm. Although these design methods have their advantages, the FEA optimization method combined with the optimal design algorithm has the highest accuracy [5]. 
The work in [5] involved multi-objective shape optimization of a PMSM based on FEA and a modified particle swarm optimization algorithm. The performance of five PMSMs with different rotor topologies were compared based on several factors including the efficiency, flux-weakening rate, and price. In [6], an optimization process for PMSM design to optimize the weight, output power, and suitability was proposed. This work included shape optimization of the permanent magnets and the rotor core using FEA with the fuzzy inference system strategy. Using a novel memetic algorithm, an optimal design was created based on FEA to minimize torque ripple in a PMSM [7]. In [8], multi-physics and multi-objective optimization of a PMSM based on FEA and an analytical magnetic model were studied.

There are two main ways to optimize design variables: either by combining the optimal algorithm with the design methods directly or by combining it with the metamodel resulting from the design of experiments (DOE). A metamodel is a mathematical model that approximates the relationships between design variables and responses. DOE is an application of statistics aimed at designing experimental methods and analyzing the results to identify relationships between design variables and responses. In the case of optimization by creating metamodels using DOE results, it is possible to predict the optimal results by analyzing the sensitivity between design variables and target goals. Moreover, the time taken for the optimization design is clear. However, the numbers of DOE and test points must be selected properly, and the metamodel must be made correctly.

Recently, there have been many studies on optimal design using the Kriging model as a metamodeling technique. The Kriging model, which is a representative of the interpolation class of models, generates an approximation function composed of a global model and the local deviation. The Kriging model is based on the empirical method applied by a South African miner named D. G. Krige. It has been widely applied in various fields since Georges Matheron, a French geologist, established its mathematical basis. Later, the Kriging model was first applied in engineering by Sacks et al. As the Kriging model is known to be suitable for representing heavy nonlinearity, it has been used to solve complicated problems of mechanical engineering [9]. Simpson et al. recommended the Kriging model as an optimal model for solving nonlinear problems with less than 50 variations [10]. In [11], the optimum design of a flux-switching PM machine was developed using the Kriging model. The cogging torque was minimized by changing the rotor pole arc and tooth width, with constraints on the average torque, torque ripple, and slot fill factor. In [12], the design and optimization of a novel wound field synchronous machine was presented. The rotor shape was optimized to improve torque and reduce torque ripple using the Kriging model and a genetic algorithm (GA). In [13], a study of a novel stator modular ring drive thruster motor was carried out. Multi-objective optimal design was performed combining FEA, a Kriging model, and non-dominated sorting GA II. In [14], an unequal air-gap model for a PMSM was proposed and it was optimized using the Kriging model and a GA. The torque ripple was reduced by $7.7 \%$ and the demagnetization temperature was increased. In the previous studies [11-14], metamodels were generated using only one Kriging model. However, because there are different suitable metamodeling methods depending on the design problem, it is necessary to apply various methods.

The use of artificial intelligence technology based on big data has been rapidly spreading and these techniques have been applied to the field of optimal design. Machine learning, a key area of artificial intelligence, is a set of learning program techniques that allow machines and computers to think and learn like humans. This process consists of accumulating data, learning rules, and predicting new data. Deep learning is a field of machine learning that utilizes multi-layered artificial neural networks. "Deep" means that the neural network has many layers. Recently, owing to the improvement of computer performance and availability of sufficient digital data, deep learning has become a rapidly developing technology. Deep learning techniques are beginning to be applied in the field of electric machines, but they are still limited to the field of fault diagnosis only [15-17]. In [15], a diagnosis algorithm for PMSMs was studied using a convolution neural network (CNN). The system, using a one-dimensional (1D)-CNN, showed $98.8 \%$ accuracy in classifying five failure modes of the PMSM. In [16], a novel motor fault diagnosis method using deep transfer learning was proposed. The $\mathrm{CNN}$ has been adopted as the basic framework for extracting multilevel functions from raw vibration 
signals. A novel deep learning framework was developed to get an accurate motor fault diagnosis using transfer learning. The test accuracy was nearly $100 \%$ for the induction motor dataset [17].

This study differs from previous ones for the following reasons. Firstly, metamodeling using a multi-layer perceptron (MLP), which is a kind of deep learning, was carried out and used for shape optimization of a PMSM. As mentioned, the use of deep learning techniques is still confined to motor fault diagnosis. Secondly, the optimal design results obtained using the MLP and the results of applying the Kriging model, which has been widely used, are compared. In the past, there have been many studies on metamodeling conducted by applying a single method, but because there is a suitable metamodel for each optimal design problem, it is necessary to create and compare metamodels in various ways. Finally, analysis was performed while changing the weighting of the multi-objective function. In the case of an optimal design with a multi-objective function, the optimal design results depend on the weighting of the multi-objective function. Therefore, this study examines the effects on the optimal design result of adjusting the weighting ratio of the multi-objective function.

In this paper, a shape optimization method for PMSMs for EVs using MLP, a type of deep learning, is proposed. To maximize the performance of a PMSM, the average torque and total harmonic distortion (THD) of the back electromotive force (EMF) were set as the multi-objective function, and the efficiency and torque ripple were set as constraints. FEA was used to improve the accuracy of the design results, and DOE was performed by adjusting the design variables. Based on the DOE results, metamodels were generated by applying the proposed MLP and the conventional Kriging model. Shape optimization was performed by applying the generated metamodels and a hybrid metaheuristic algorithm (HMA), and the results were compared. FEA for the optimized model was conducted to verify the validity of the shape optimization design results. To verify the structural stability, mechanical stress analysis was performed on the optimal model. In addition, the changes in the PMSM performance were examined while changing the weighting of the multi-objective function. The overall process of this study is represented in the flowchart shown in Figure 1.

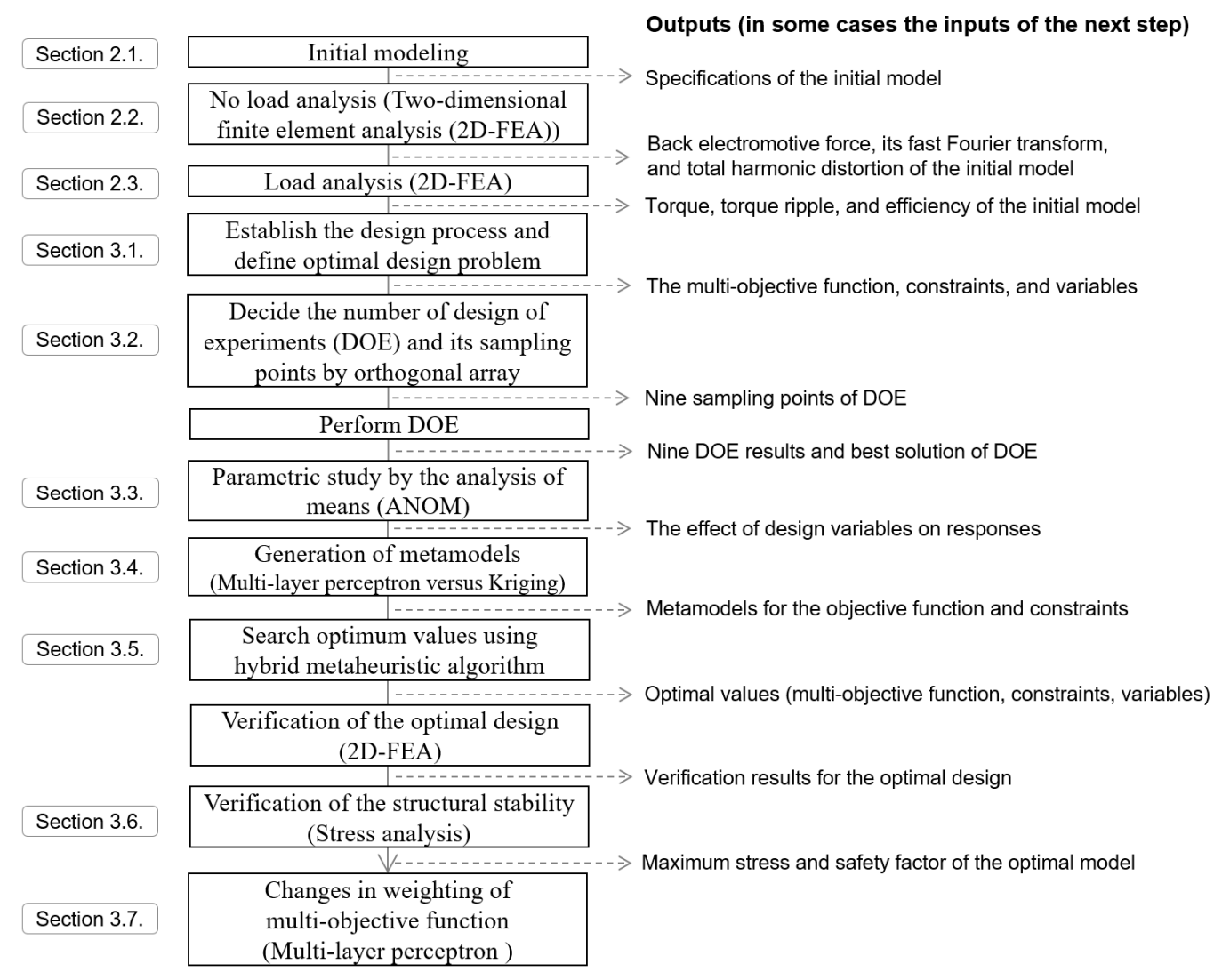

Figure 1. Flowchart of the overall research procedure. 


\section{Finite Element Analysis}

Renault's Twizy model is popular among small EVs. In this model, a $13 \mathrm{~kW}$ induction motor with a torque of $57 \mathrm{~N} \cdot \mathrm{m}$ at $2100 \mathrm{rpm}$ was used. With reference to the model, the target specification of this study was determined. A PMSM was selected as the initial model, and the target output power was set at $15 \mathrm{~kW}$, which should provide a torque of $60 \mathrm{~N} \cdot \mathrm{m}$ at $2387 \mathrm{rpm}$.

\subsection{Initial Model}

The shape and specifications of the $15 \mathrm{~kW}$ PMSM for a small EV are shown in Figure 2 and Table 1, respectively. The rotor has eight poles and consists of $\mathrm{V}$-shaped $\mathrm{NdFeB}$ magnets for magnetic flux concentration. The stator has 36 slots and is wound in distributed windings. To reduce core loss, a 0.35 -mm-thick electrical steel sheet is used as the core material. The rotor core has notches to reduce the cogging torque.

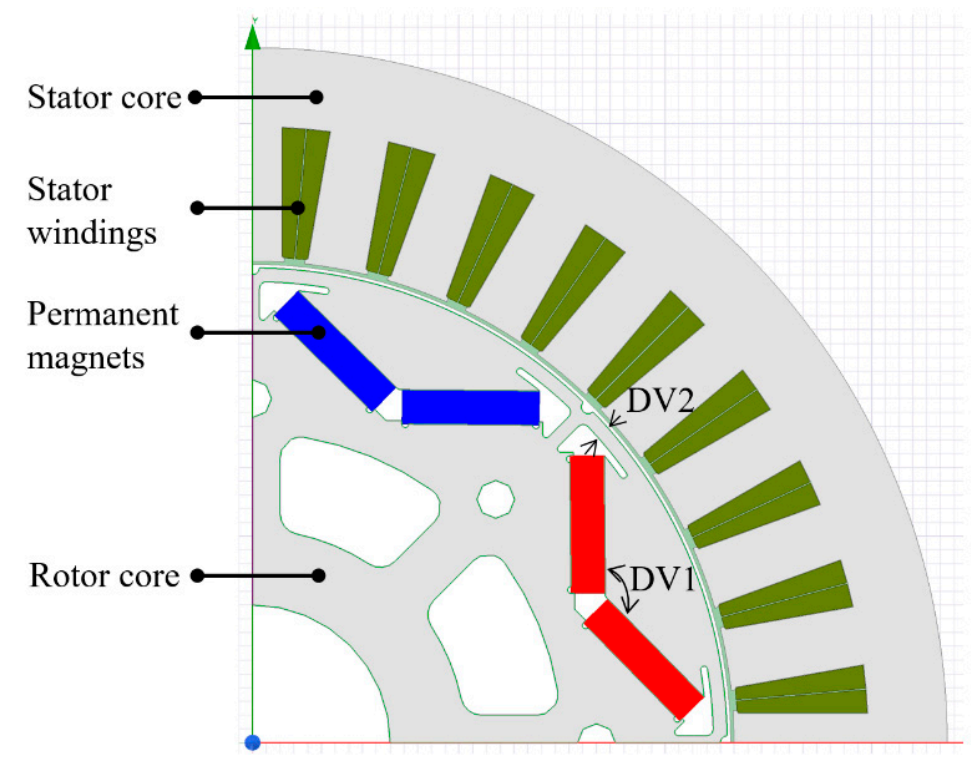

Figure 2. Shape and design variables of the initial permanent magnet synchronous motor model (1/4 model).

Table 1. Specifications of analysis model.

\begin{tabular}{cccc}
\hline & Items & Unit & Value \\
\hline \multirow{2}{*}{ Required spec. } & Max. output power & $\mathrm{kW}$ & 15 \\
& Max. torque & $\mathrm{N} \cdot \mathrm{m}$ & 60 \\
& Rated speed & $\mathrm{rpm}$ & 2387 \\
\hline \multirow{2}{*}{ Material } & Electrical steel & - & 35PN210 (POSCO) \\
& Permanent Magnet & - & $\mathrm{N} 38 \mathrm{UH}$ \\
\hline \multirow{2}{*}{ Electrical } & Continuous current & $\mathrm{A}_{\mathrm{rms}}$ & 146 \\
& Current phase angle & $\circ$ & 25 \\
& Winding spec. & - & $\varnothing 0.9,11$ turns (3strand) \\
\hline \multirow{3}{*}{ Mechanical } & Number of poles and slots & ea & $8 / 36$ \\
& Outer diameter of stator & $\mathrm{mm}$ & 202 \\
& Inner diameter of stator & $\mathrm{mm}$ & 140 \\
& Outer diameter of rotor & $\mathrm{mm}$ & 138 \\
& Inner diameter of rotor & $\mathrm{mm}$ & 40 \\
& Air-gap & $\mathrm{mm}$ & 1 \\
& Lamination & $\mathrm{mm}$ & 45 \\
\hline Thermal & Reference temperature & ${ }^{\circ} \mathrm{C}$ & 20 \\
\hline
\end{tabular}




\subsection{No Load Analysis}

FEA was conducted to increase the accuracy of the analysis. When the rotor of the PMSM rotates under the no-load condition, back EMF is induced in the stator winding. THD is an important factor in electrical equipment and power systems. The THD can be obtained by adding the harmonic components to the fundamental wave components of the voltage or current [18]. A higher THD increases the core loss in electric machines, which can reduce the efficiency and generate excessive heat. The calculated THD of the back EMF of the initial model is $3.519 \%$.

\subsection{Load Analysis}

To find the input current condition, a torque analysis was performed based on changes in the current as a function of the current phase angle, as shown in Figure 3. The current condition that satisfies the rated torque of $60 \mathrm{~N} \cdot \mathrm{m}$ at the rated speed of $2387 \mathrm{rpm}$ requires that the RMS value of the current is $146 \mathrm{~A}$ and the current angle is $25^{\circ}$. Load analysis was performed under this current condition. The average torque was found to be $60.05 \mathrm{~N} \cdot \mathrm{m}$ and the torque ripple was $6.951 \%$ of the torque. The output power of the motor can be calculated as the product of torque and angular velocity. If the mechanical loss is ignored, total losses of the motor are the sum of the core loss and the copper loss. The efficiency can be calculated from the output power and total losses of the motor. The efficiency of the initial model was calculated to be $91.43 \%$.

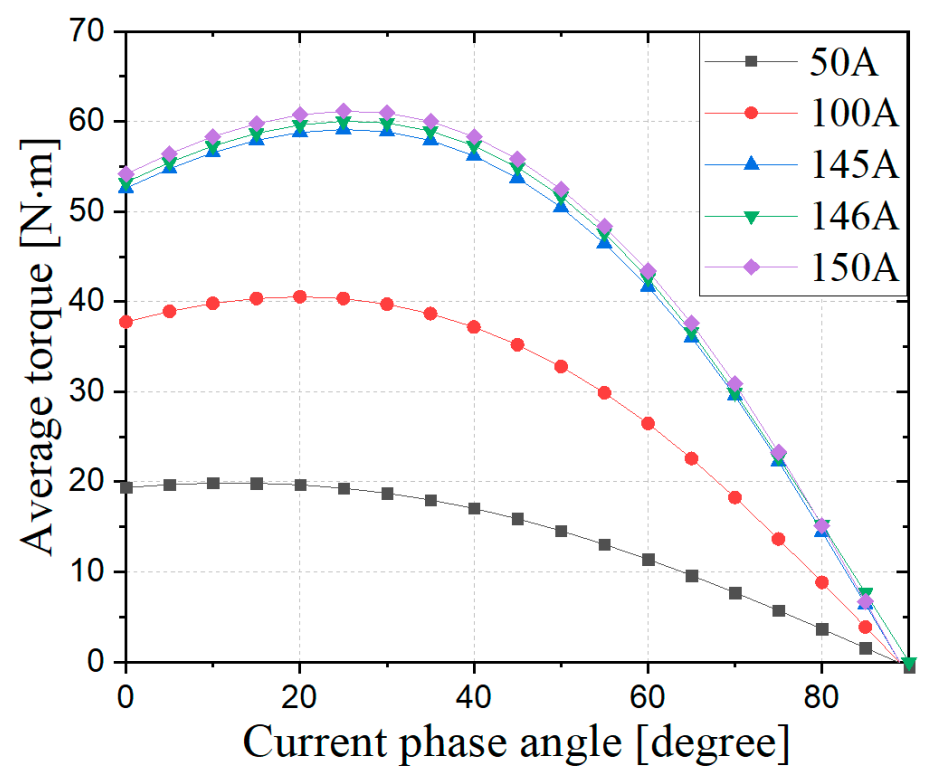

Figure 3. Torque as a function of the changes in current and current phase angle.

\section{Design Optimization}

\subsection{Design Process}

The process used for shape optimization of the PMSM is shown in Figure 4. First, a multi-objective function, constraints, and design variables were established to achieve the required target specifications. The multi-objective function was set as Equation (1) to satisfy the objective of simultaneously maximizing torque and minimizing the THD of the back EMF. Because the efficiency and torque ripple should be maintained properly even if the objective function is achieved, the constraints were set as shown in Equation (2). The torque ripple was set below $10 \%$, which is an acceptable level for a traction motor for an EV [19]. The design variables were chosen to be the inner angle between the V-shaped permanent magnets (PM) and the rib thickness of the rotor to satisfy the multi-objective function and constraints, as shown in Equation (3). The sampling points of the design variables for the DOE were 
determined using the orthogonal array $(\mathrm{OA})$ technique. DOE was performed by using two-dimensional (2D)-FEA to ensure the accuracy of the experimental results. Based on the DOE results, metamodeling of the multi-objective function and constraints was performed using MLP, a type of deep learning. Metamodeling using Kriging, which has been widely used in the past, was also carried out for comparative verification of the results obtained using the MLP technique. Metamodeled results were combined with the optimal algorithm, HMA, to obtain the optimal geometric design parameter values.

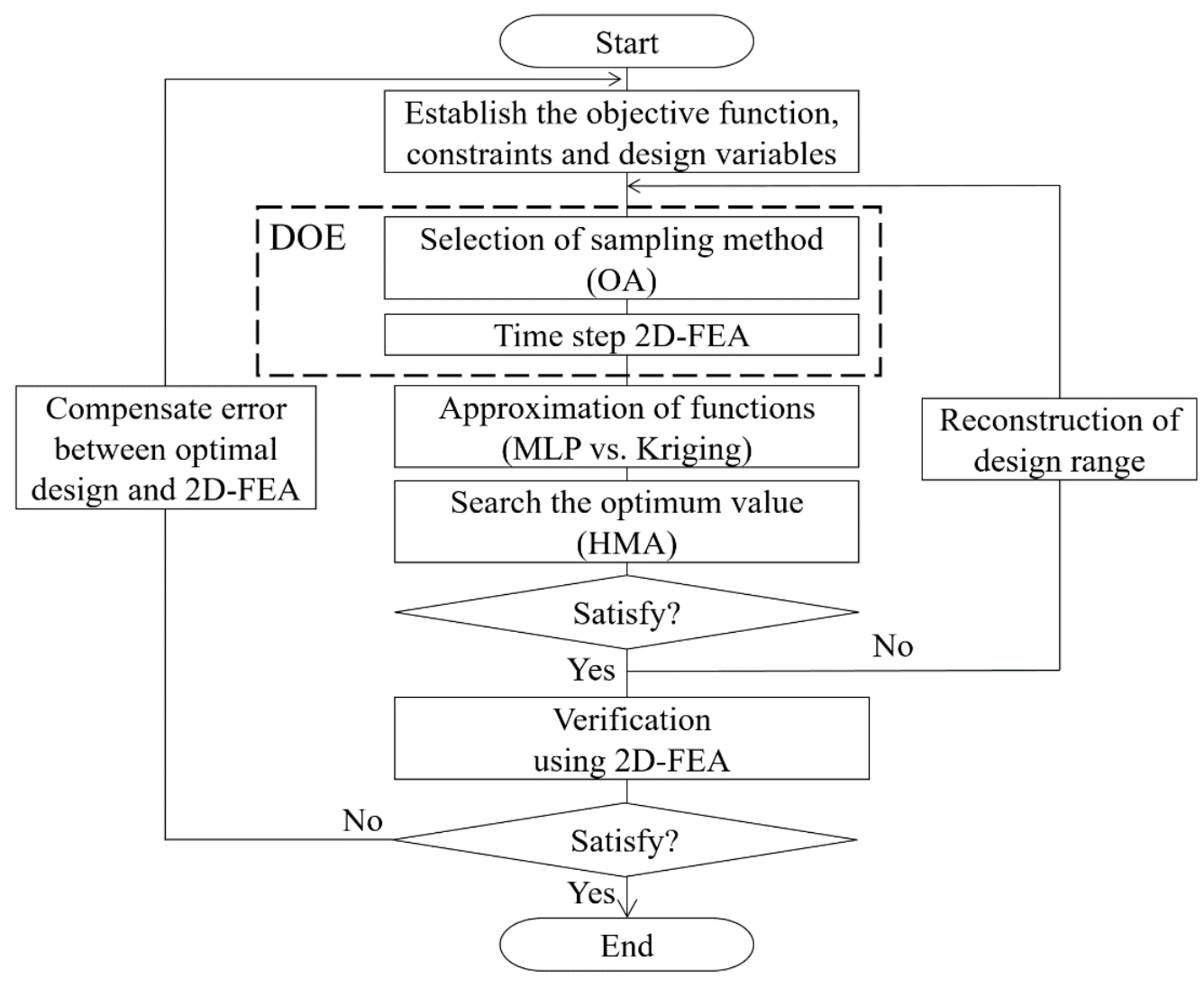

Figure 4. Optimization design process.

Objective function

Maximize the average torque

Minimize THD of the Back EMF

Constraints

$$
\begin{gathered}
\text { Efficiency } \geq 91.43 \% \\
\text { Torque ripple } \leq 10 \%
\end{gathered}
$$

Design variables

$$
\begin{gathered}
130^{\circ} \leq \mathrm{DV} 1 \text { (Inner angle between permanent magnets) } \leq 142^{\circ} \\
1 \mathrm{~mm} \leq \mathrm{DV} 2 \text { (Rib thickness) } \leq 2 \mathrm{~mm}
\end{gathered}
$$

\subsection{Design of Experiments}

DOE refers to a method of designing a set of experiments to solve a design problem and includes an experimental method, a data acquisition method, and a data analysis method. If the DOE works well, the maximum amount of information is obtained from the minimum number of experiments. The number of experiments should be selected according to the number of design variables and levels. Here, the number of design variables was two and the number of levels was three; therefore, the total number of 
experiments was nine. The OA technique was applied to determine the sampling points of the DOE. $\mathrm{OA}$ is designed to reduce the number of experiments in the full factorial design and is one of the fractional factorial design methods. It is a technique to reduce the number of experiments by confounding negligible interactions within the main factorial design with the main effect. In addition, this technique satisfies orthogonality, and it can be easily used because the relative coordinates of the experimental points are already determined. To improve the accuracy of the analysis, each experiment was conducted using 2D-FEA and the results of DOE are shown in Table 2. The DOE results show that torque improvement is relatively easy, but it is difficult to improve the THD of the back EMF. Further improvement is difficult because the initial model's THD value is already low enough at $3.519 \%$. At test point 7 , the torque increased to $63.411 \mathrm{~N} \cdot \mathrm{m}$, but the THD increased to $4.082 \%$ compared to that in the initial model. Therefore, the eighth experiment with a $2.86 \%$ torque improvement is the optimal experimental point.

Table 2. DOE results. DOE: design of experiments.

\begin{tabular}{ccccccccccc}
\hline Items & Unit & DOE1 & DOE2 & DOE3 & DOE4 & DOE5 & DOE6 & DOE7 & DOE8 & DOE9 \\
\hline $\begin{array}{c}\text { Inner angle between } \\
\text { permanent magnets }\end{array}$ & $\circ$ & 130 & 130 & 130 & 136 & 136 & 136 & 142 & 142 & 142 \\
Rib thickness & $\mathrm{mm}$ & 1 & 1.5 & 2 & 1 & 1.5 & 2 & 1 & 1.5 & 2 \\
\hline $\begin{array}{c}\text { Average torque } \\
\text { Total harmonic distortion of }\end{array}$ & $\mathrm{N} \cdot \mathrm{m}$ & 62.791 & 61.169 & 59.714 & 63.147 & 61.514 & 60.046 & 63.411 & 61.764 & 60.302 \\
the back EMF & $\%$ & 4.066 & 3.616 & 3.547 & 4.075 & 3.599 & 3.519 & 4.082 & 3.590 & 3.488 \\
$\quad \begin{array}{c}\text { Efficiency } \\
\text { Torque ripple }\end{array}$ & $\%$ & 91.766 & 91.571 & 91.385 & 91.809 & 91.614 & 91.429 & 91.839 & 91.645 & 91.463 \\
& $\%$ & 7.836 & 7.342 & 6.928 & 7.816 & 7.325 & 6.951 & 7.767 & 7.298 & 6.962 \\
\hline
\end{tabular}

\subsection{Parametric Study}

To analyze the effect of the design variables on the multi-objective function and constraints, the analysis of means (ANOM) was performed, as shown in Figure 5. Consequently, it can be seen that the rib thickness has a greater influence on the multi-objective function and constraints compared to the inner angle between the PM. In particular, the standard deviation of torque according to the change of rib thickness was 1.264, which suggests the largest effect on torque among the multi-objective function and constraints.

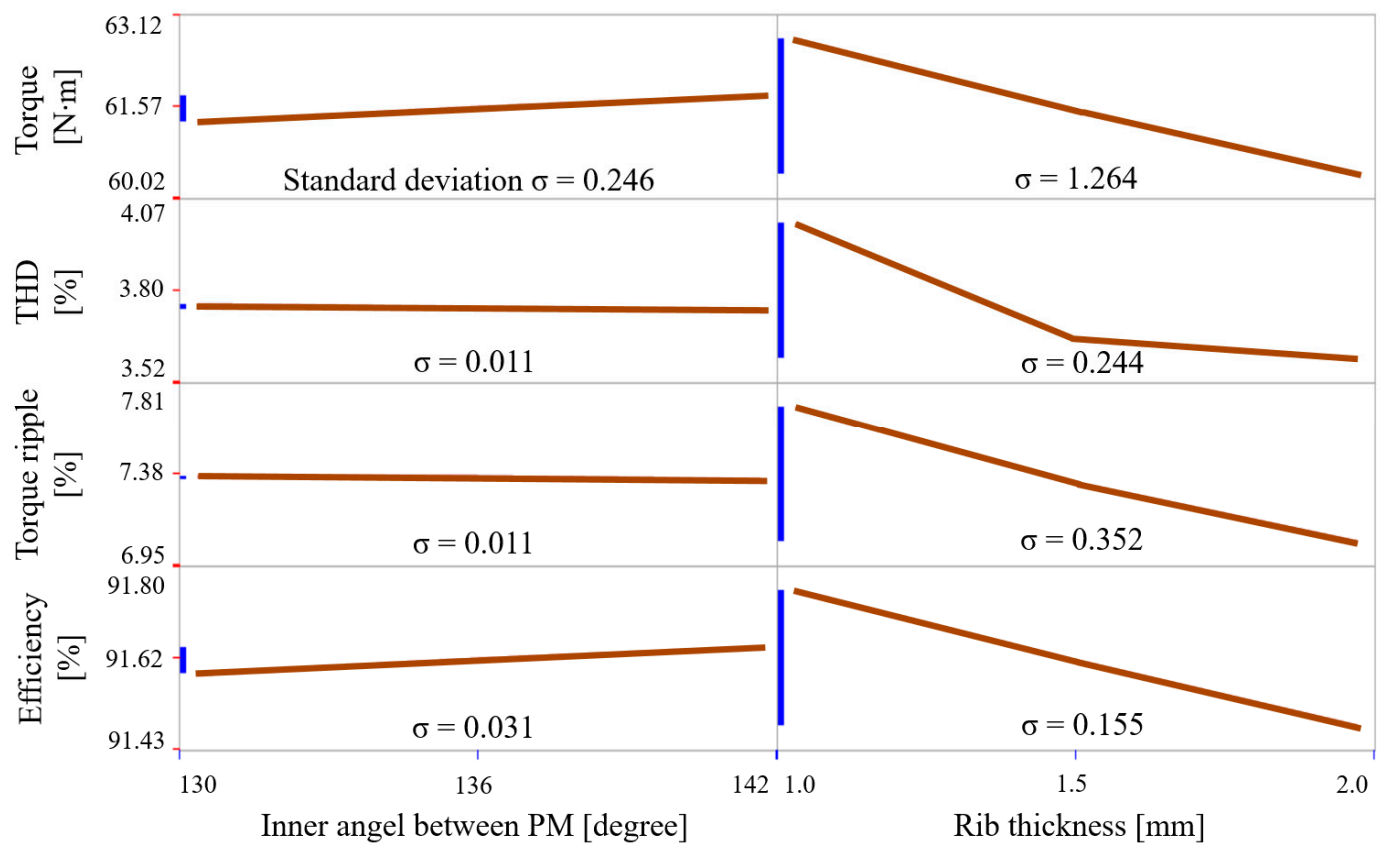

Figure 5. The analysis of means results. 


\subsection{Metamodeling}

Based on the DOE results, metamodels for the multi-objective functions and constraints were generated. Metamodels were generated using the proposed MLP and the conventional Kriging model. These metamodels can be classified as a regression model and an interpolation model, respectively. Regression models, e.g., polynomial regression (PR), radial basis function regression (RBFr), ensemble of decision trees (EDT), and MLPs, smoothen the noise in the data because they do not pass through the test points exactly. Therefore, this type of model is useful for real experiments with random errors. MLP is a type of deep learning algorithm and has the advantage of being able to represent nonlinear relationships between the input and output variables [20]. Neural networks can learn complex patterns using neurons that mathematically transform data. A neuron, also called a perceptron, is a mathematical function. One or more inputs are multiplied by weights, and the results are then passed to a nonlinear function known as the activation function for output. One layer that consists of several neurons is called a hidden layer. The number of hidden layers and the number of neurons that make up each hidden layer are determined by the design problem, as shown in Figure 6.

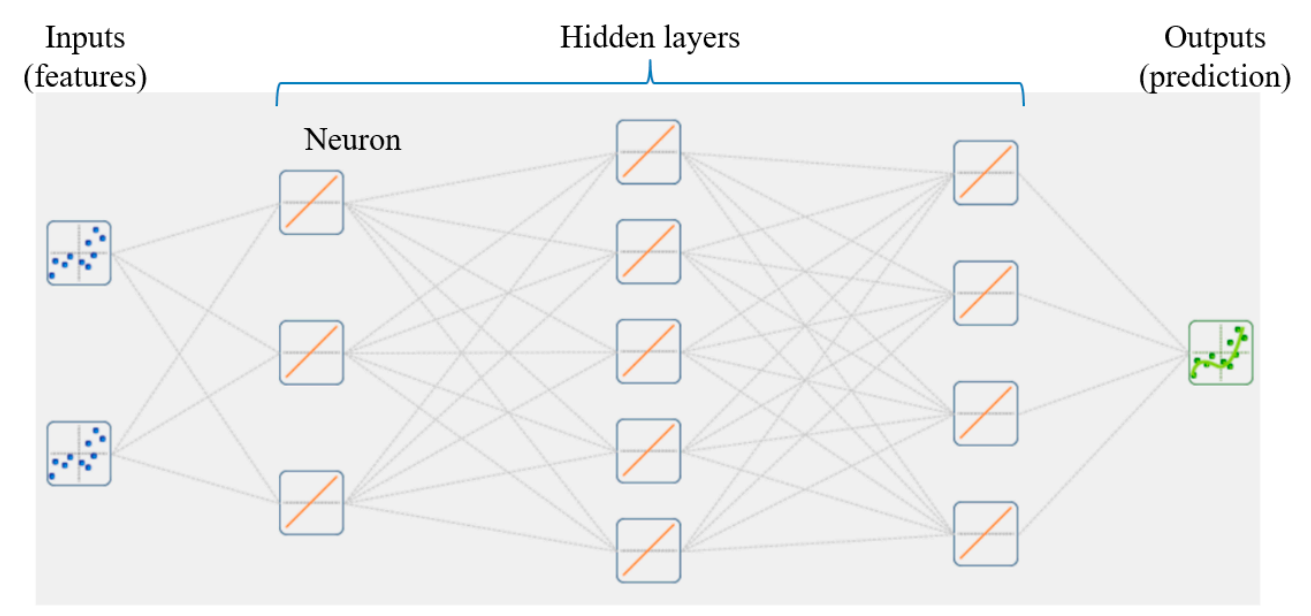

Figure 6. Structure of MLP.

In contrast, interpolation models, e.g., Kriging model and radial basis function regression interpolation ( $\mathrm{RBFi})$, are well suited for function approximation using analytical results without random errors because these models pass through the test points exactly. The estimated equation of the Kriging model was defined to eliminate bias and thereby minimize error variance [21]. The Kriging model is a weighted linear combination as follows:

$$
z *=\sum_{i=1}^{n} \lambda_{i} z_{i}
$$

where $z^{*}$ is an estimated point using the Kriging model, $n$ is the total number of experiments, $\lambda_{i}$ is the weight value function, and $z_{i}$ is the experimental point.

Metamodeling was carried out using a software package for optimal design called PIAnO. Metamodeling using MLP requires the selection of hyperparameters such as the number of hidden layers and neurons, activation functions, and learning rates. Hyperparameters can also be selected manually, but it is difficult to find the optimal value and these values do not reflect any prior knowledge gained during the investigation. Therefore, hyperparameters were automatically selected to maximize the predictive performance of the MLP using the 'hyperparameter optimization' function provided by PIAnO [22]. Limited-memory Broyden-Fletcher-Goldfarb-Shanno (L-BFGS) was used as the optimization algorithm for the automatic selection of hyperparameters, as this algorithm is suitable for large-scale numerical optimization [23]. The metamodeling results for the multi-objective function 
and constraints using MLP are shown in Figure 7. The metamodels for the torque and THD of the back EMF set by the multi-objective function are shown in Figure 7a,b. The number of hidden layers of the MLP for the torque was seven and the number of neurons of each layer was between two and five. The number of hidden layers for the THD of the back EMF was one, and the number of neurons was three. The activation function of neurons was also automatically selected by the 'hyperparameter optimization' function provided by PIAnO, and the icons of the neurons in Figure 7 represent the selected activation function [22]. The activation functions of neurons for the torque and THD of the back EMF were hyperbolic tangent and sigmoid functions, respectively. Torque ripple is calculated from the average and the peak-to-peak values of the torque. Efficiency is also calculated from the mechanical output and losses. Because the torque ripple and efficiency were calculated using the FEA results, they are not suitable for direct metamodeling. Therefore, metamodels for the peak-to-peak value of the torque and core loss were generated, as shown in Figure $7 \mathrm{c}, \mathrm{d}$. The number of hidden layers of the core loss model was three and the number of neurons of each layer was between three and five. The number of hidden layers for the peak-to-peak torque model was two, and the number of neurons of each layer was between three and four. The activation functions of the neurons for the core loss and peak-to-peak torque models were linear and hyperbolic tangent functions, respectively. Using these metamodeling results, the torque ripple and efficiency were calculated using VBScript, as shown in Figure 8.

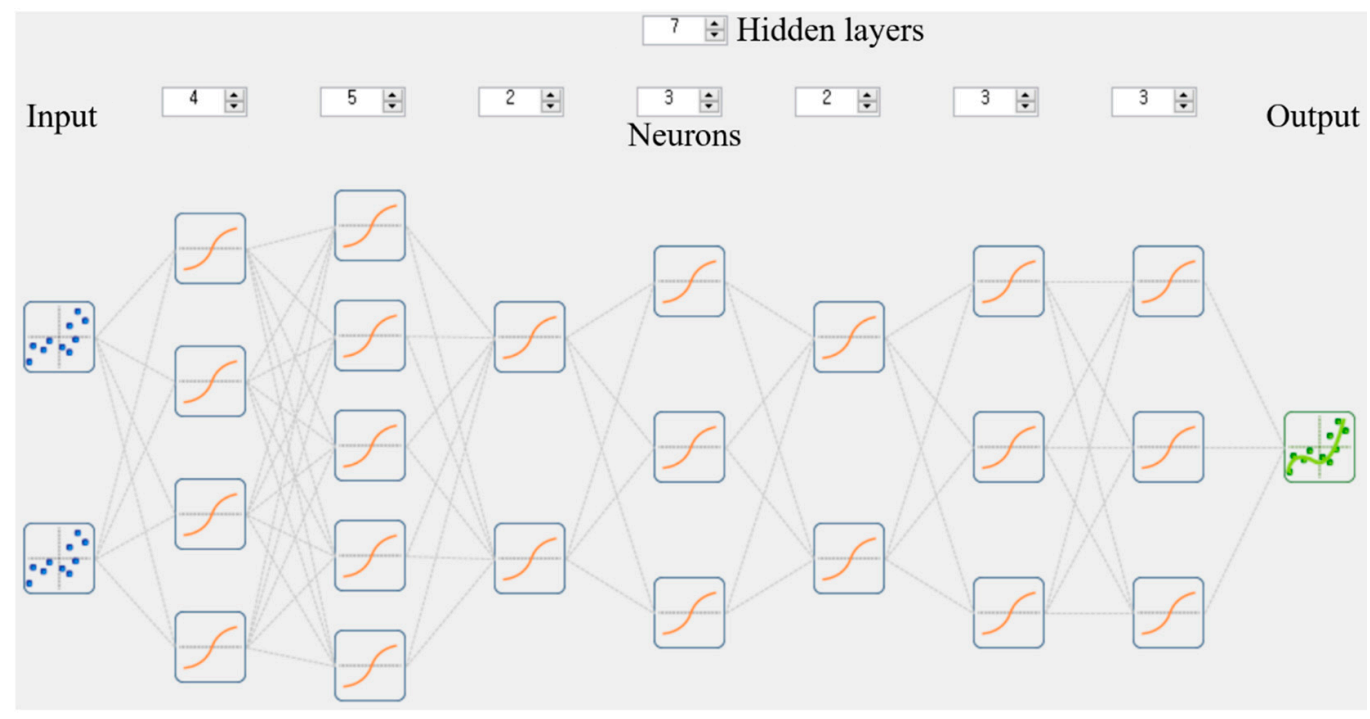

(a)

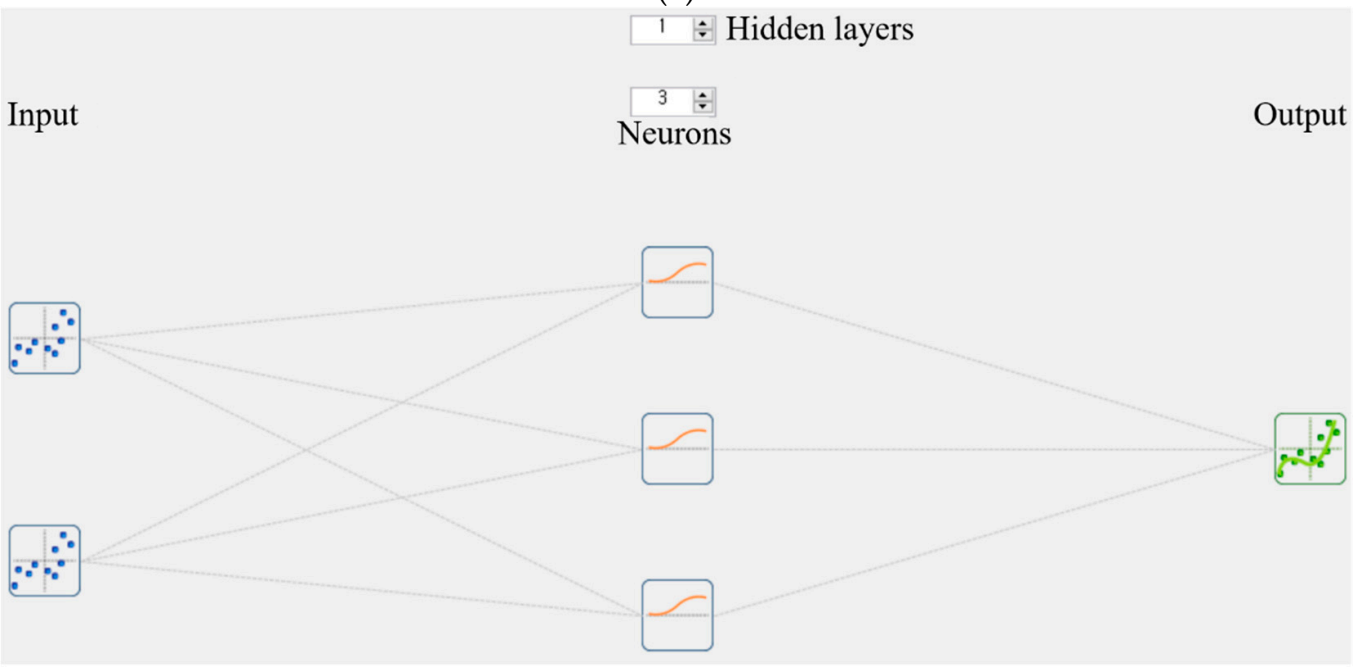

(b)

Figure 7. Cont. 


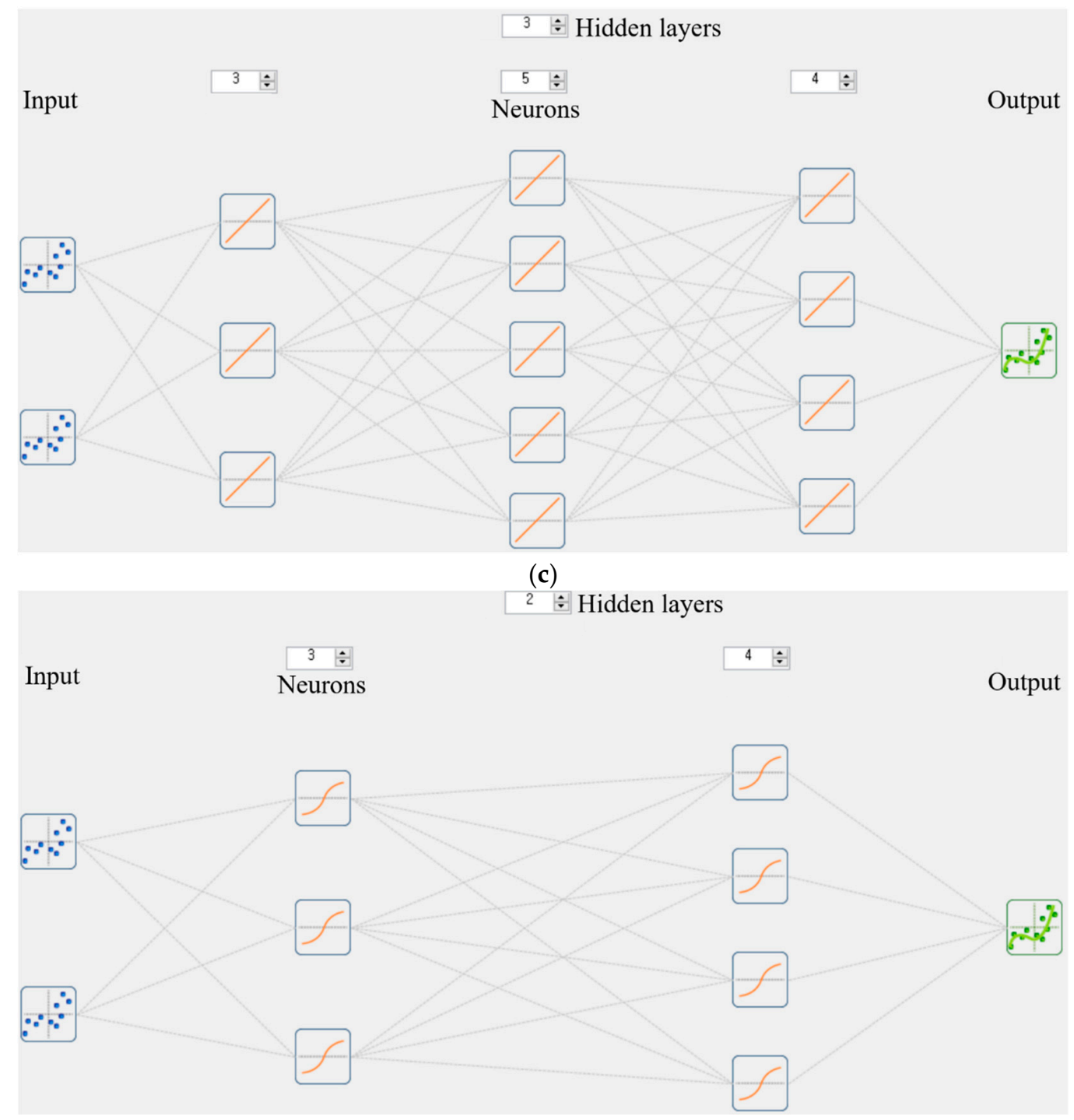

(d)

Figure 7. Metamodels using MLP: (a) torque; (b) THD of back EMF; (c) core loss; (d) peak to peak torque.

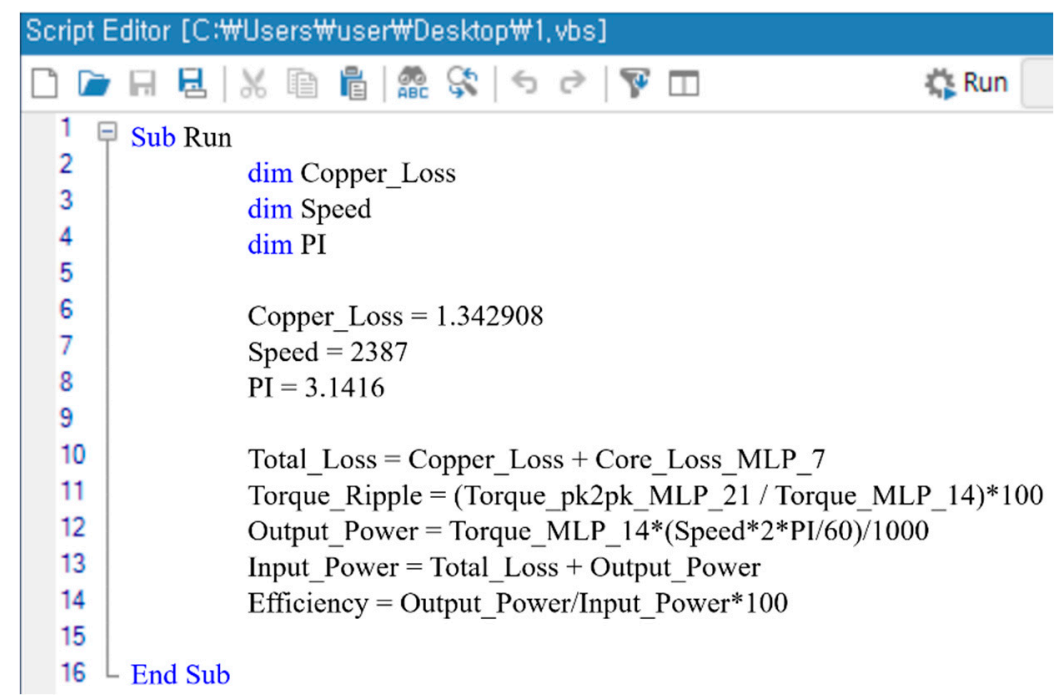

Figure 8. VBScript used to calculate torque ripple and efficiency using metamodels. 


\subsection{Design Optimization Based on Metamodel}

Optimal design was carried out using the metamodels generated using an MLP and Kriging, respectively. HMA was used as the optimal design algorithm, and the ratio of the weighting between the average torque and the THD of the back EMF was set to 0.5:0.5. The HMA, a global optimization algorithm, was used for the optimal design based on the metamodel. The HMA was proposed in 2016 by Park [24]. The HMA can determine the global optimum faster than other global optimizers owing to the combined advantages of improved constrained differential evolution (rank-iMDDE) and modified cuckoo search (MCS). Table 3 shows the optimum design results. When Kriging was used, the average torque was improved by $1.3 \%$ compared to the initial model, while when MLP was used, it was improved by $2.2 \%$. For the THD of the back EMF and constraints, the Kriging and MLP results were almost equal. Using MLP required somewhat more function calls than using Kriging, but convergence time decreased by five seconds. The optimal design results confirm that the proposed metamodeling-based optimal design results obtained using MLP had better prediction performance than the conventional Kriging model. In addition, FEA for the optimal model was conducted to verify the optimal design results. Therefore, the validity of the optimal design result was verified. Figure 9 shows the torque waveforms of the initial model and the optimal model. The average torque of the optimal model was improved by $2.2 \%$, but the torque ripple was equivalent. The improvement of the torque characteristics may be due to the smooth change of the magnetic flux flow, as shown in Figure 10. The improvements in the magnetic flux flow and the change in reluctance can be attributed to the increase in the back EMF and torque [19]. Owing to the improvements in the flux flow, the back EMF of the optimum model was $34.0 \mathrm{~V}$, which represents an improvement of $4.3 \%$ compared to the $32.6 \mathrm{~V}$ of the initial model, as shown in Figure 11a. The results of harmonic analysis of the back EMF of both models were almost similar, as shown in Figure 11b, and its THD values were also almost the same, as described in Table 3.

Table 3. Optimization results.

\begin{tabular}{ccccccc}
\hline \multirow{2}{*}{ Items } & Unit & $\begin{array}{c}\text { Initial } \\
\text { (FEA) }\end{array}$ & $\begin{array}{c}\text { Optimal } \\
\text { (Kriging) }\end{array}$ & $\begin{array}{c}\text { Optimal } \\
\text { (MLP) }\end{array}$ & $\begin{array}{c}\text { Optimal } \\
\text { (FEA) }\end{array}$ \\
\hline \multirow{2}{*}{ Design variables } & Inner angle between PM & $\circ$ & 136 & 142 & 142 & 142 \\
& Rib thickness & $\mathrm{mm}$ & 2.0 & 1.8 & 1.6 & 1.6 \\
\hline \multirow{5}{*}{ Design results } & Average torque & $\mathrm{N} \cdot \mathrm{m}$ & 60.05 & 60.83 & 61.36 & 61.46 \\
& THD of the back EMF & $\%$ & 3.519 & 3.497 & 3.526 & 3.521 \\
& Efficiency & $\%$ & 91.43 & 91.53 & 91.59 & 91.61 \\
& Torque ripple & $\%$ & 6.951 & 7.053 & 7.196 & 7.236 \\
\cline { 2 - 7 } & Function calls & - & - & 1942 & 2387 & - \\
& Convergence time & $\mathrm{sec}$ & - & 27 & 22 & - \\
\hline
\end{tabular}

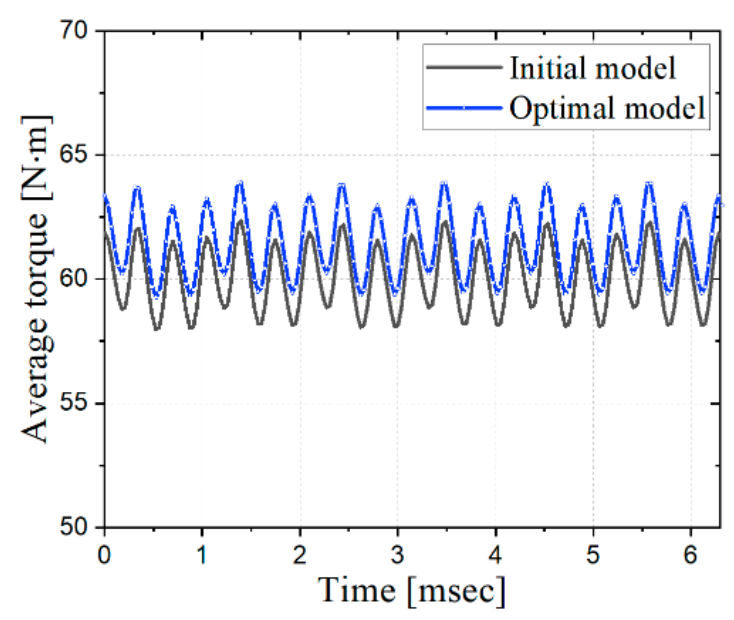

Figure 9. Torque waveforms. 


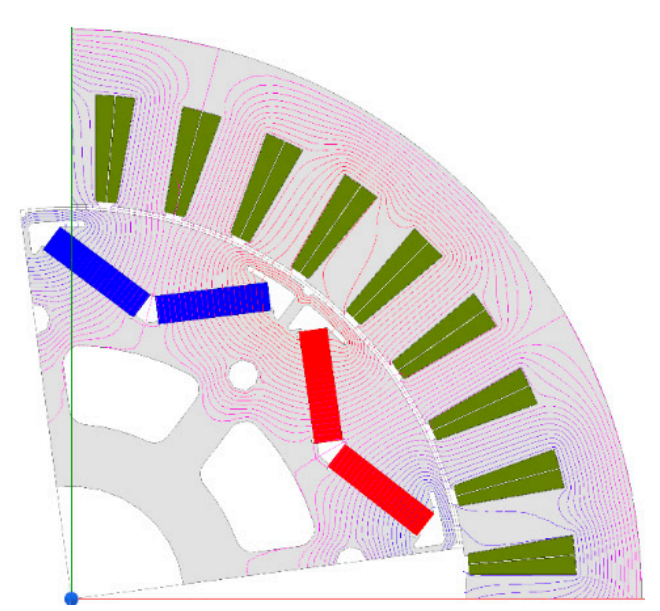

(a)

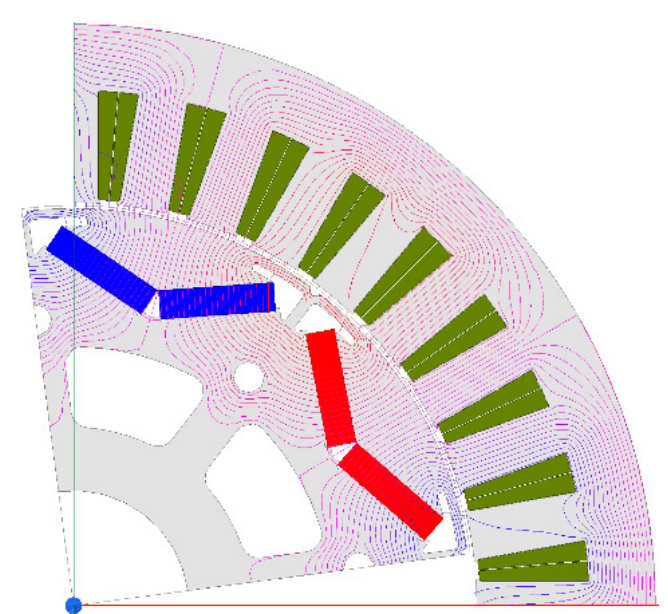

(b)

Figure 10. Flux distribution under the no load condition: (a) initial model; (b) optimal model.

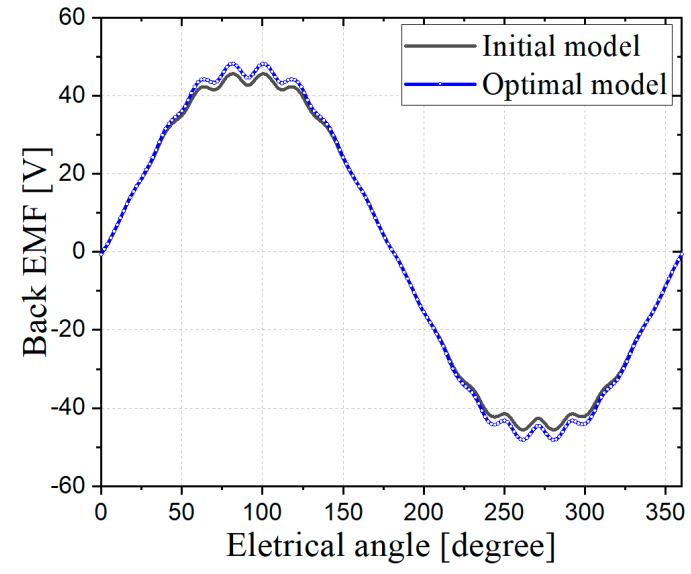

(a)

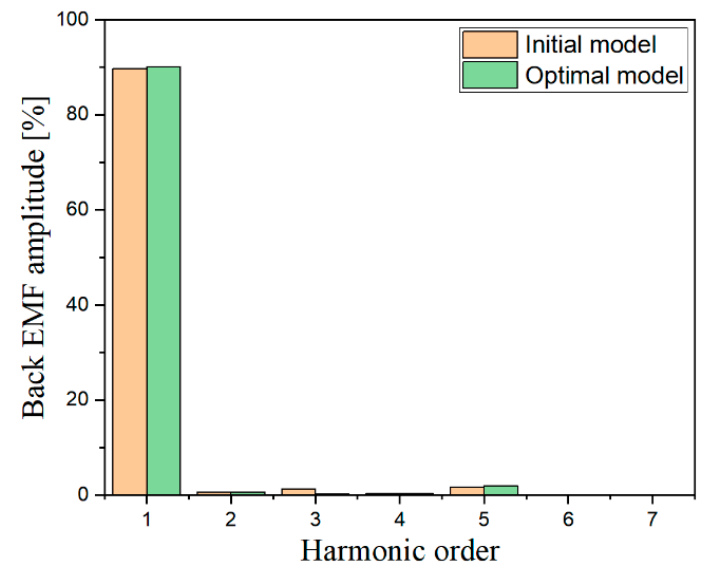

(b)

Figure 11. The back EMF of the initial model and optimal model: (a) waveform; (b) harmonic.

\subsection{Mechanical Stress Analysis}

It is important to check the mechanical stress of the rotor when the geometry of the rotor core and the positions of the permanent magnets change. When the permanent magnets inserted inside the rotor rotate at high speed, a strong centrifugal force is generated; thus, the strength of the rotor core should be verified. Therefore, mechanical stress analysis of the optimal model was performed using ANSYS Mechanical. The material properties of the electrical steel and permanent magnets used for the analysis are shown in Table 4. The maximum stress of the optimal model was $88.493 \mathrm{MPa}$ at $8000 \mathrm{rpm}$, which was lower than the tensile yield strength of the electrical steel, and it occurred in the protrusion of the rotor core used to fix the permanent magnets, as shown in Figure 12. The calculated safety factor was 4.69 , which proves the feasibility of the proposed shape optimization.

Table 4. Material properties for mechanical stress analysis.

\begin{tabular}{cccc}
\hline Items & Unit & Electrical Steel (35PN210) & Permanent Magnet (N38UH) \\
\hline Density & $\mathrm{kg} / \mathrm{m}^{3}$ & 7600 & 7500 \\
Poisson's ratio & - & 0.3 & 0.24 \\
Young's modulus & $\mathrm{GPa}$ & 170 & 160 \\
Tensile yield strength & $\mathrm{MPa}$ & 415 & 80 \\
\hline
\end{tabular}




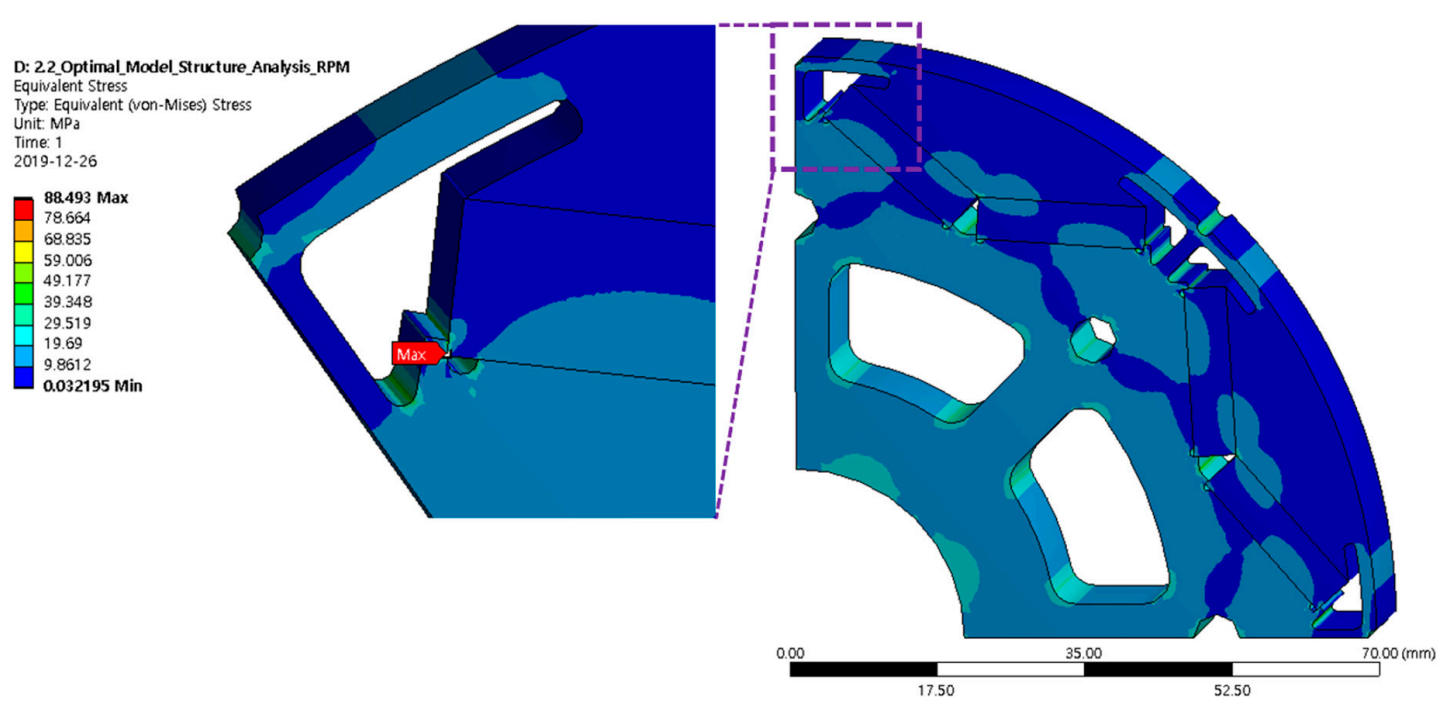

Figure 12. Mechanical stress generated in the rotor of the optimal model.

\subsection{Change in Weighting of Multi-Objective Function}

Table 5 shows the optimal design results according to changes in the weighting between the average torque and THD of the back EMF, based on the metamodel generated using the MLP. To improve the THD, the weighting ratio of the average torque and the THD was adjusted to 0.3:0.7. However, the THD was slightly improved by $0.04 \%$, while the average torque was significantly reduced by $1.74 \%$. Therefore, it was confirmed that the improvement of THD is difficult compared to that of the average torque. In addition, even if the weighting of the multi-objective function was adjusted, the improvement of THD was still difficult.

Table 5. Optimization results according to the weighting change.

\begin{tabular}{ccccccc}
\hline \multicolumn{2}{c}{ Items } & Unit & Initial (FEA) & \multicolumn{3}{c}{ Optimal (MLP) } \\
\hline Weighting ratio (Average torque: & THD of the back EMF) & - & - & $0.5: 0.5$ & $0.4: 0.6$ & $0.3: 0.7$ \\
\hline \multirow{2}{*}{ Design variables } & Inner angle between PM & $\circ$ & 136 & 142 & 142 & 142 \\
& Rib thickness & $\mathrm{mm}$ & 2.0 & 1.6 & 1.6 & 2.0 \\
\hline \multirow{3}{*}{ Design results } & Average torque & $\mathrm{N} \cdot \mathrm{m}$ & 60.05 & 61.36 & 61.36 & 60.29 \\
& THD of the back EMF & $\%$ & 3.519 & 3.526 & 3.526 & 3.488 \\
& Efficiency & $\%$ & 91.43 & 91.59 & 91.59 & 91.46 \\
& Torque ripple & $\%$ & 6.951 & 7.196 & 7.196 & 6.963 \\
\hline
\end{tabular}

\section{Conclusions}

This paper presents the shape optimization of a PMSM using an MLP, a kind of deep learning method. To maximize the performance of the PMSM, the average torque and THD of the back EMF were set as the multi-objective function, and efficiency and torque ripple were set as constraints. FEA was used to improve the accuracy of the design results, and DOE was performed based on changes of the design variables. As a result of the parametric study by ANOM, the rib thickness was determined to be a design variable that greatly affects the responses. Based on the DOE results, metamodels were generated by applying the proposed MLP technique and the conventional Kriging technique. Shape optimization was performed by applying the generated metamodels and HMA, and the results were compared. Average torque was increased by $2.2 \%$ with the MLP and by $1.3 \%$ with the Kriging model. In both cases, the THD of the back EMF, efficiency, and torque ripple were nearly equal. Therefore, in this design model, the metamodel using the MLP achieved better prediction performance than the conventional Kriging model. The back EMF was also increased by $4.3 \%$ compared with the initial model owing to the improvement of the magnetic flux flow. The structural stability of the optimal model was verified by 
mechanical stress analysis. Optimal design analysis was carried out by changing the weighting of the multi-objective function, and it was confirmed that the THD of the back EMF was difficult to improve compared to the average torque. This study shows that an MLP, a type of deep learning technique, is useful as a metamodeling technique for shape optimization of PMSM. It is expected that MLPs can be used for the shape optimization of various electric machines in the near future.

Funding: This study was supported by research fund from Honam University, 2018 and the National Research Foundation of Korea (NRF) grant funded by the Korea government (MSIT). (No. NRF-2018R1C1B5046117).

Acknowledgments: The author express gratitude to PIDOTECH and FRONTIS for their technical support.

Conflicts of Interest: The author declares no conflict of interest.

\section{References}

1. Hong, G.; Wei, T.; Ding, X. Multi-objective optimal design of permanent magnet synchronous motor for high efficiency and high dynamic performance. IEEE Access 2018, 6, 23568-23581. [CrossRef]

2. Feng, G.; Lai, C.; Kar, N.C. An analytical solution to optimal stator current design for PMSM Torque ripple minimization with minimal machine losses. IEEE Trans. Ind. Electron. 2017, 64, 7655-7665. [CrossRef]

3. Dang, L.; Bernard, N.; Bracikowski, N.; Berthiau, G. Design optimization with flux weakening of high-speed PMSM for electrical vehicle considering the driving cycle. IEEE Trans. Ind. Electron. 2017, 64, 9834-9843. [CrossRef]

4. Ilka, R.; Alinejad-Beromi, Y.; Yaghobi, H. Techno-economic design optimisation of an interior permanent-magnet synchronous motor by the multi-objective approach. IET Electr. Power Appl. 2018, 12, 972-978. [CrossRef]

5. Song, T.; Zhang, Z.; Liu, H.; Hu, W. Multi-objective optimisation design and performance comparison of permanent magnet synchronous motor for EVs based on FEA. IET Electr. Power Appl. 2019, 13, 1157-1166. [CrossRef]

6. Krasopoulos, C.T.; Beniakar, M.E.; Kladas, A.G. Multicriteria PM motor design based on ANFIS evaluation of ev driving cycle efficiency. IEEE Trans. Electron. 2018, 4, 525-535. [CrossRef]

7. Lee, J.H.; Kim, J.W.; Song, J.Y.; Kim, Y.J.; Jung, S.Y. A novel memetic algorithm using modified particle swarm optimization and mesh adaptive DIRECT search for PMSM design. IEEE Trans. Magn. 2016, 52, 7001604. [CrossRef]

8. Zhao, W.; Wang, X.; Gerada, C.; Zhang, H.; Liu, C.; Wang, Y. Multi-physics and multi-objective optimization of a high speed PMSM for high performance application. IEEE Trans. Magn. 2018, 54, 8106405. [CrossRef]

9. Lee, S.H.; Kim, H.Y.; Oh, S.I. Cylindrical tube optimization using response surface method based on stochastic process. J. Mater. Process. Technol. 2002, 130-131, 490-496. [CrossRef]

10. Simpson, T.W.; Peplinski, J.D.; Koch, P.N.; Allen, J.K. Metamodels for computer-based engineering design: Survey and recommendations. Eng. Comput. 2001, 17, 129-150. [CrossRef]

11. Kwon, J.W.; Lee, J.H.; Zhao, W.L.; Kwon, B.I. Flux-switching permanent magnet machine with phase-group concentrated-coil windings and cogging torque reduction technique. Energies 2018, 11, 2758. [CrossRef]

12. Chai, W.P.; Lipo, T.A.; Kwon, B.I. Design and optimization of a novel wound field synchronous machine for torque performance enhancement. Energies 2018, 11, 2111. [CrossRef]

13. Li, Y.K.; Song, B.W.; Mao, Z.Y.; Tian, W.L. Analysis and optimization of the electromagnetic performance of a novel stator modular ring drive thruster motor. Energies 2018, 11, 1598. [CrossRef]

14. You, Y.M.; Chung, D.W. Optimal design of a permanent magnet synchronous motor to improve torque and demagnetization characteristics. J. Magn. 2017, 22, 423-429. [CrossRef]

15. Kao, I.H.; Wang, W.J.; Lai, Y.H.; Perng, J.W. Analysis of permanent magnet synchronous motor fault diagnosis based on learning. IEEE Trans. Instrum. Meas. 2019, 68, 310-324. [CrossRef]

16. Xiao, D.Y.; Huang, Y.X.; Zhao, L.J.; Qin, C.Q.; Shi, H.T.; Liu, C.L. Domain adaptive motor fault diagnosis using deep transfer learning. IEEE Access 2019, 7, 80937-80949. [CrossRef]

17. Shao, S.Y.; McAleer, S.; Yan, R.Q.; Baldi, P. Highly accurate machine fault diagnosis using deep transfer learning. IEEE Trans. Ind. Inform. 2019, 15, 2446-2455. [CrossRef]

18. Narayanan, G.; Ranganathan, V.T. Analytical evaluation of harmonic distortion in PWM AC drives using the notion of stator flux ripple. IEEE Trans. Power Elctron. 2005, 20, 466-474. [CrossRef] 
19. Chau, K.T. Electric Vehicle Machines and Drives: Design, Analysis and Application, 1st ed.; Wiley-IEEE: Hoboken, NJ, USA, 2015; p. 243.

20. Zhang, L.; Tian, F. Performance study of multilayer perceptrons in a low-cost electronic nose. IEEE Trans. Instrum. Meas. 2014, 36, 1670-1679. [CrossRef]

21. Sacks, J.; Welch, W.J.; Mitchell, T.J.; Wynn, H.P. Design and analysis of computer experiments. Stat. Sci. 1989, 4, 409-435. [CrossRef]

22. PIDOTECH Inc. PIAnO User's Manuals and Tutorials; PIDOTECH Inc.: Seoul, Korea, 2019.

23. Ge, F.; Ju, Y.; QI, Z.; Lin, Y. Parameter estimation of a gaussian mixture model for wind power forecast error by riemann L-BFGS optimization. IEEE Access 2018, 6, 38892-38899. [CrossRef]

24. Park, K.B. An Efficient Hybrid Metaheuristic Algorithm for Solving Constrained Global Optimization Problems. Ph.D. Thesis, Hanyang University, Seoul, Korea, February 2016.

(C) 2020 by the author. Licensee MDPI, Basel, Switzerland. This article is an open access article distributed under the terms and conditions of the Creative Commons Attribution (CC BY) license (http://creativecommons.org/licenses/by/4.0/). 